\title{
New Directions for Preserving Intangible Cultural Heritage Through the Use of Mobile Technologies
}

Konstantinos Papangelis

Xi'an Jiaotong-Liverpool University

Suzhou, Jiangsu, P.R. China

K.papangelis@xjtlu.edu.cn

\section{Alan Chamberlain}

University of Nottingham

Jubilee Campus, Wollaton Road,

Nottingham, NG8 1BB, UK

Alan.Chamberlain@nottingham.ac.uk

\section{Hai-Ning Liang}

Xi'an Jiaotong-Liverpool University

Suzhou, Jiangsu, P.R China

HaiNing.Liang@xjtlu.edu.cn

\begin{abstract}
While many cultural heritage projects currently exist, few explore the full potential of mobile technologies as a mechanism to explore intangible heritage as a way to preserve culture. This paper outlines three distinct areas necessary for the design, development and application of mobile technologies within this domain. We represent these as: a) The documentation of traditions within their unique context, as articulated by the represented community-co-curated; b) The translation of traditions and their modes of expression into emerging technology-based designs; c) Co-design and ethnography as approaches to build meaningful mobile experiences.
\end{abstract}

\section{Author Keywords}

Intangible Cultural Heritage; Mobile Technologies;

Cultural Preservation; Indigenous; Design.

\section{ACM Classification Keywords}

H.1.2 User/Machine Systems

\section{Introduction}

In an effort to prevent the disappearance of indigenous heritage, many digital preservation projects are in 


\section{The Kasidawen dance of the Qiang}

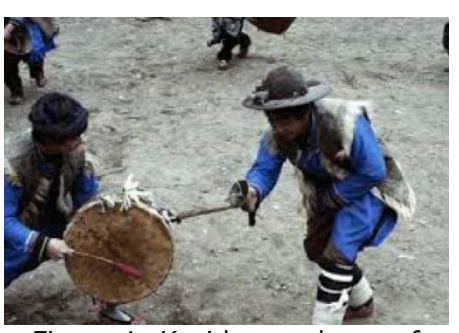

Figure 1: Kasidawen dance of

warriors preparing for battle.

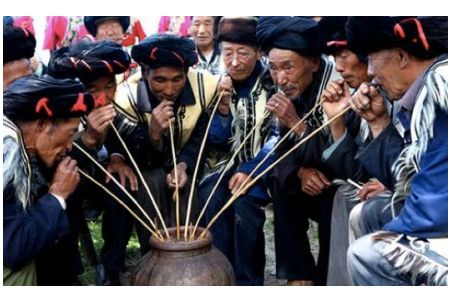

Figure 2: Qiang performing predance rituals

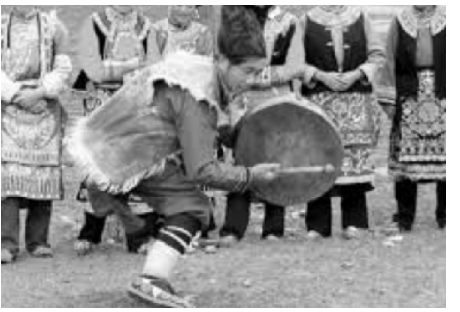

Figure 3: Kasidawen dance

demonstration for the Qiang

youth.

Source: Beijing Cultural Heritage Protection Center progress. An example of this is the Traditional Arts \& Ethnology Centre at Luang Prabang Laos; the centre recorded the Iu Mien New Year celebration and the Kim Di Mun ordination using high-definition digital audio and video in order that these ceremonies might be preserved. There are concerns that as the older generation passes away, the cultural traditions and rituals associated with these celebrations may be lost forever. The digitization of intangible cultural heritage through digital media and mobile technologies can potentially preserve them for future generations, but still key questions arise about the presentation of such content, and the modalities and mechanisms through which these are mediated.

\section{Digital preservation of Intangible Cultura Heritage through Mobile Technologies}

A variety of digital preservation projects have mainly used digital technology and mixed media to preserve and promote the cultures of indigenous people (e.g. see $[1,2])$. The use of such media potentially means that access can be globally. However, an examination of such projects informs us that the vast majority of them are primarily focused upon documentation. Thus far mobile technologies have played a small role in capturing the intangible indigenous cultural heritage; this is in part due to researchers mainly focusing on capturing heritage 'snapshots' and not engaging with the people that they are documenting for extended periods; and in part because of the changing nature of such traditions. It is our belief that mobile devices can play an important role in not only augmenting the reach of such projects, but also engaging segments of indigenous groups, which otherwise may not engage with the preservation of such traditions.
Involving the indigenous people in the process of developing mobile technologies may include developing strategies to enable people to utilise (or appropriate already existing) mobile technologies, create digital toolsets that allow modification and customization for their particular content and context, and explore the design and development of technology according to their goals and ways of thinking. This enables indigenous peoples to play a role in the development of emerging technology and contemporary modes of cultural expression as well as co-developing technologies in relation to the understanding of those cultures

\section{The proposed three step process for preserving intangible cultural heritage}

The goal of preservation, promotion and growth of intangible heritage of indigenous cultures requires a 3phase strategy for digital preservation projects through mobile technologies, including 1) Documentation, 2) Translation into technology, and 3) A set of principles for the development of new mobile technologies.

What follows is an outline of this three-phase strategy applied to an envisaged project relating to the preservation of the Kasidawen dance of the Qiang Ethnic Group in China. The Qiang people (In Chinese: 危族; pinyin: Qiāng zú) are one of the 56 ethnic groups recognized in China, and live in the mountainous region in the northwestern part of Sichuan on the eastern edge of the Tibetan Plateau (see Figure 4).

The Kasidawen (In Chinese: 美族舞) dance is prevalent mainly in the Heishui River valley of Aba Prefecture in Sichuan province. "Kasida" means armor, and "Wen" 


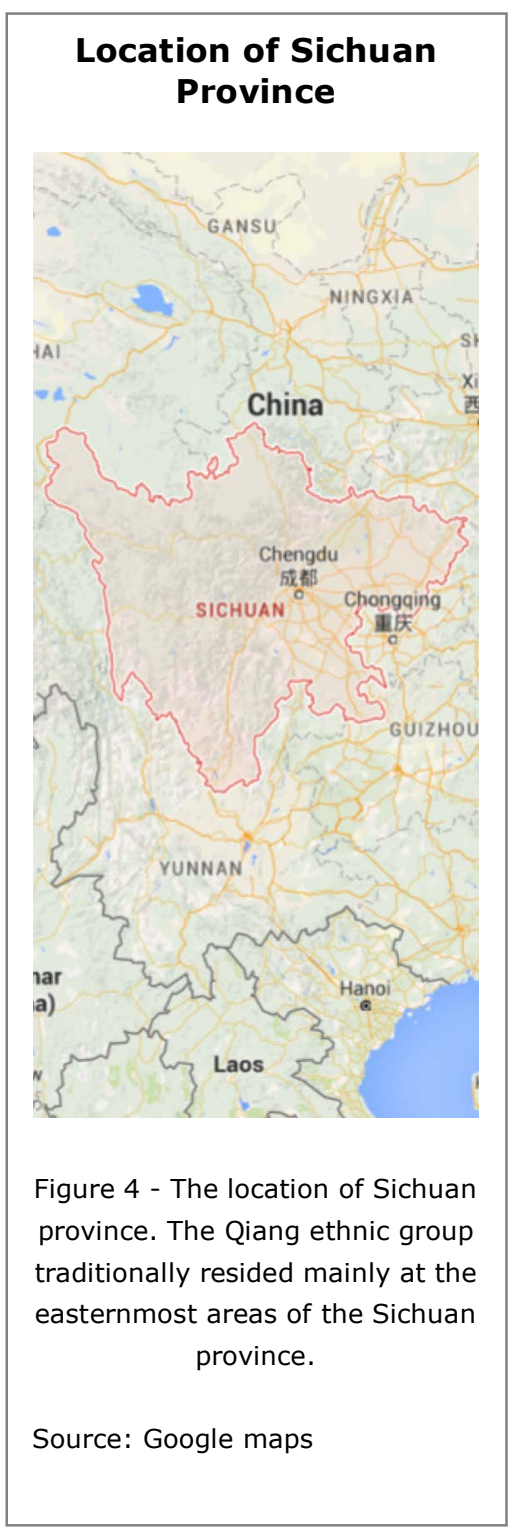

"to wear" as the dance is performed wearing armors. Kasidawen was originally performed prior to hunting, before battles in the past, and now at various festivals. The Kasidawen varies from place to place in Heshui Country in terms of both the content and form (see figure 1,2 and 3 for more details). For example, in the Zhuba Villave of Zhawo Township it represents hunting; in the E'en Village of Hongyan Township it represents battles; and in Wigu Township it covers topics such as, lovers saying goodbye, or conveying a feeling of mourning for fallen warriors. The Qian culture is predominantly oral in nature, and is passed down the generations through their oral traditions. With the rapid growth of modern economy in China, many Qian people are migrating to cities leaving behind rural communities, and languages, customs and rituals of their ancestors behind. In order to co-create a digital strategy for digital preservation of the Kasidawen dance we present the following strategy, focusing upon the use of mobile technologies as a mechanism that can be used to capture, curate and mediate heritage-based content.

\section{The documentation of traditions within their}

\section{unique context}

This would involve the creation of materials and the documentation of both tangible and intangible culture that has significance to the communities that are represented. This can include videos, audio recordings, interviews, animation, booklets, websites, pamphlets, roles and step-by-step instructions on how to perform the Kasidawen dance. This content might be accessed in location using smart phones such that it might have more relevance to the setting; it may be provided in a multilingual format and could even be accessed at festivals and other events where the dance is performed. Although there is some existing documentation of intangible heritage, these, 1) usually only provide snapshot of the intangible heritage that they focus upon - it is not ongoing and therefore, 2) they do not provide the natural evolutionary timeline of the tradition, because the people documenting are not usually part of the community that is being documented, they 3 ) focus only on major/popular variations of the dance and usually ignore minor variation or minority groups within the indigenous community 4) Additionally the content is difficult to accesses by those communities and by other people (in context).

Translations of modes of expression of traditions into contemporary emerging technologies

This phase involves exploring the role of the Kasidawen's dance in relation to the technology and the habits of the Qiang today. This may involve for example interviews/focus groups with various Qiang groups. Initial explorations may reveal that socially meditated mobile technology can be utilized at both ends directly (e.g. illustrating the dance, enabling the capturing of aspects of the dance "in the wild"), or enable discussions (e.g. utilizing mobile technologies to view various aspects of the dance) throughout the process. The main focus in this step is to present and enable a constructive dialogue regarding the Kasidawen dance within contemporary media and tools, but also to understand how these can be used to explore the social purposes of the dance in various contexts and create new understandings.

Application principles for the development of new mobile technologies 


\section{Acknowledgements}

Dr. Chamberlain's input into this research was supported through the following EPSRC grant: Living with Digital Ubiquity (EP/M000877/1).
The customs and traditions of indigenous cultures are able to produce and enhance technologies to be used by these communities. Different ways of thinking, modes of learning, symbols, visualization, and the goals underlying less dominant cultures can richly contribute to the design and application of new technologies. As such the main focus of this step is to involve the indigenous population in the co-creation process of technology according to their lives, practices, ways of living, thinking and their traditions. In this instance this phase involves co-creating with the Qiang mobile systems that take into account the nature of the intangible heritage we are interested in preserving, and co-translating this in a set of high-level guidelines that will guide the co-creation process. This can be achieved through extensive ethnography and various direct and indirect methods of knowledge acquisition. For example, the Kasidawen dance has a social nature, and as such within the Qiang population special focus should be given to a design that enables the 'social' nature to be interpreted by the users, perhaps facilitating conversation and enabling the Qiang to capture aspects around the dance, their life and enriching these by using mobile technologies. By pairing designers and developers with Kasidawen dancers we may allow the often-intangible aspects of the tradition to find new potential in the form of technological innovation.

\section{Discussion and Conclusion}

There are limitations and risks inherent in taking such approaches. For example, certain traditions are tied to specific roles within a community, so questions of sensitivity, privacy and ownership must be taken into account. There are also concerns in respect of altering the meaning of the tradition by using mobile media. For example, the Kasidawen dance is an intimate social experience, and no amount of smart design will replace the experience of a Qiang or outsider participating in or observing the dance. Another aspect we have to consider is that these projects will be more accessible to technologically enabled societies or segments of the indigenous population than to the technologically underserved areas, that could produce the content, and while digitization through mobile technologies might help narrow the gap caused by the digital divide between various communities, the question of which society benefits more must be considered. The digitization of heritage through the use of mobile technologies can raise risks as well as open opportunities. This short paper presented a 3-phase model to involve the communities in the digitization process through the use of mobile technologies. These three phases aim to ensure that preservation of intangible heritage does not imply nostalgic snapshots of marginalized cultures, but involves a meaningful and dynamic ever-evolving co-exploration (and even further development) of the ideas, goals, effects, and outputs of the cultural tradition.

\section{References}

1. Byamugisha, J., Sitbon, L. \& Brereton, M. (2014). Cultural and Linguistic Localization of Games to Bridge the Digital and Cultural Divide nn Indigenous Population. Proc. of the 26th Australian Computer-Human Interaction Conference on Designing Futures the Future of Design - OzCHI '14.

2. Mulhollann, E., Thorpe, K. \& Gardiner, G. (2011). Connecting Research Data and Indigenous Communities. Proc. of the 11th Int'l ACM/IEEE joint conference on Digital libraries - JCDL '11. 